Proceeding Series of the Brazilian Society of Computational and Applied Mathematics

\title{
Validação de modelo entomológico com dependência da pluviosidade para o Aedes aegypti
}

\section{Lillia dos Santos Barsante, Fernanda Soares Veríssimo,2 Rodrigo Tomás Nogueira Cardoso, José Luiz Acebal ${ }^{4}$}

Pós-Graduação em Modelagem Matemática e Computacional, CEFET, Belo Horizonte, MG Carlos Alexandre Silva ${ }^{5}$

Departamento de Informática, IFMG, Sabará, MG

\section{Álvaro Eduardo Eiras ${ }^{6}$}

Departamento de Parasitologia, Instituto de Ciências Biológicas, UFMG, Belo Horizonte, MG

Resumo. Neste trabalho validamos a população de fêmeas do Aedes aegypti obtida via modelo entomológico matemático computacional com influência da pluviosidade acumulada semanalmente, utilizando dados amostrais de Índice Médio de Fêmeas Aedes em uma região. Foi observado coincidências na posição dos picos e vales apenas ao longo do horizonte chuvoso.

Palavras-chave. Dengue, Aedes aegypti, Modelo, Pluviosidade, Índice Médio de Fêmeas Aedes.

\section{Introdução}

Atualmente tornou-se crescente o estudo de modelos matemáticos e computacionais que buscam descrever, analisar e/ou controlar a dinâmica espacial dos vetores de diversas arboviroses, tais como a dengue, malária, leishmaniose, esquistossomose e doença de Chagas. Dentre estas arboviroses, destaca-se a dengue, onde mais de 3,6 bilhões de pessoas vivem em áreas de risco à doença [12].

Dentre as espécies de fêmeas de mosquitos do gênero Aedes que podem servir como vetores desta doença, destaca-se a do Aedes aegypti. Este vetor também é responsável por outras arboviroses, tais como o vírus do chikungunya, da febre amarela urbana e do zika vírus. O ciclo de vida completo deste vetor é composto pelas fases: imatura (ovos, larvas

\footnotetext{
${ }^{1}$ lilliabarsante@gmail.com

${ }^{2}$ fsv.13.fv@gmail.com

${ }^{3}$ rodrigoc@des.cefetmg.br

${ }^{4}$ acebal@dppg.cefetmg.br

${ }^{5}$ carlos.silva@ifmg.edu.br

${ }^{6}$ alvaro.eiras@gmail.com
} 
e pupas) e alada (mosquitos adultos). A fase imatura tem duração média entre 8-13 dias e a fase alada tem duração média entre 30-45 dias [2].

A dinâmica sazonal do vetor da dengue está associada ao aumento da temperatura, variações na pluviosidade e umidade do ar [11]. De acordo com [6], a pluviosidade, a temperatura e outros fatores, afetam a sobrevivência, a reprodução, a dispersão e a densidade do vetor da dengue. Segundo [5] a pluviosidade aumenta consideravelmente a quantidade de criadouros disponíveis para o desenvolvimento da fase imatura e também gera condições ideais para o desenvolvimento da fase alada do vetor, além de exercer grande influência na determinação do período de ocorrência da dengue. A influência de variáveis metereológicas na dinâmica das populações do ciclo de vida do $A$. aegypti e na transmissão da dengue têm sido abordados em diversos trabalhos como ( [3], [4], [7]).

Neste trabalho, validaremos um modelo entomológico matemático computacional que descreve a dinâmica da população do ciclo de vida do Ae. aegypti sob influência da pluviosidade acumulada semanalmente $(p)$ com dados amostrais de Índice Médio de Fêmeas Aedes(IMFA), capturadas pela armadilha MosquiTRAP ${ }^{\circledR}$, no horizonte que corresponde às semanas epidemiológicas 12 a 52 do ano de 2009 e 1 a 51 do ano de 2010, da cidade de Sete Lagoas (Minas Gerais, Brasil).

\section{Modelo Matemático}

O modelo entomológico matemático computacional utilizado neste trabalho foi proposto por [1] e está ilustrado na Figura 1.

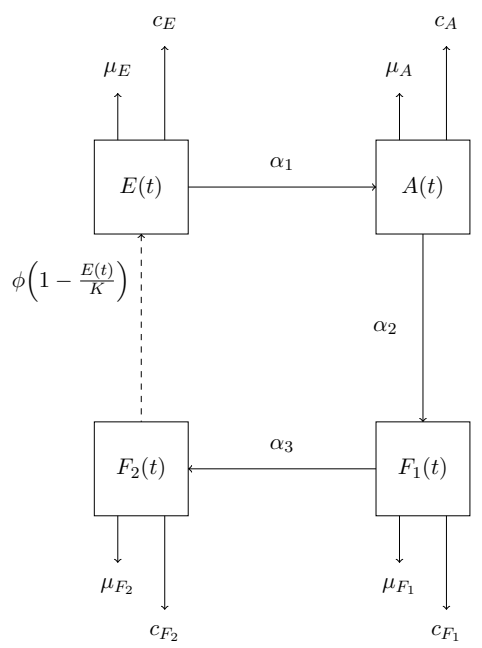

Figura 1: Diagrama ilustrando a dinâmica das populações do ciclo de vida do Aedes aegypti com quatro compartimentos: ovos $E(t)$, aquática (larvas + pupas) $A(t)$, fêmeas pré-repasto sanguíneo $F_{1}(t)$, e fêmeas pós-repasto sanguíneo $F_{2}(t)$. Os parâmetros $\alpha_{i}$ com $i=1,2,3$ representam os índices de desenvolvimento do vetor: $\alpha_{1}$ - ovos para aquática, $\alpha_{2}$ - aquática para fêmeas pré-repasto sanguíneo, e $\alpha_{3}$ - fêmeas prérepasto sanguíneo para fêmeas pós-repasto sanguíneo; $\phi$ é a taxa de oviposição; $K$ é a capacidade do meio; $\mu$ e $c$ representam, respectivamente, a taxa de mortalidade natural e a taxa de mortalidade adicional específica em cada compartimento. 
O sistema de equações diferenciais não lineares que representa esta dinâmica é dado por:

$$
\left\{\begin{array}{l}
d E / d t=\phi(p)\left(1-\frac{E(t)}{K}\right) F_{2}(t)-\left(\alpha_{1}(p)+\mu_{E}(p)+c_{E}(t)\right) E(t), \\
d A / d t=\alpha_{1}(p) E(t)-\left(\alpha_{2}(p)+\mu_{A}(p)+c_{A}(t)\right) A(t), \\
d F_{1} / d t=\alpha_{2}(p) A(t)-\left(\alpha_{3}(p)+\mu_{F_{1}}(p)+c_{F_{1}}(t)\right) F_{1}(t), \\
d F_{2} / d t=\alpha_{3}(p) F_{1}(t)-\left(\mu_{F_{2}}(p)+c_{F_{2}}(t)\right) F_{2}(t), \\
\phi, \alpha_{1}, \alpha_{2}, \alpha_{3}, \mu_{E}, \mu_{A}, \mu_{F_{1}}, \mu_{F_{2}}, K, c_{E}, c_{A}, c_{F_{1}}, c_{F_{2}} \geq 0, \forall p, t \in \mathbb{R}_{+} .
\end{array}\right.
$$

Observe que as populações do modelo (1) foram dadas em termos relativos à capacidade do meio, $K$. Este modelo é não autônomo, uma vez que os seus coeficientes dependem da pluviosidade, e estes são funções variantes no tempo. No entanto, o modelo se comporta como uma sucessão de modelos autônomos com coeficientes definidos pela pluviosidade acumulada semanalmente da cidade em estudo, apresentando dois pontos de equilíbrio, $P_{0}$ e $P_{1}$, como segue abaixo:

$$
P_{0}=\left\{\begin{array}{l}
E^{*}=0 \\
A^{*}=0 \\
F_{1}^{*}=0 \\
F_{2}^{*}=0
\end{array} \quad \text { e } \quad P_{1}=\left\{\begin{array}{l}
E^{* *}=K\left(1-\frac{1}{R_{M}}\right) \\
A^{* *}=\frac{\alpha_{1}}{\left(\alpha_{2}+\mu_{A}+c_{A}\right)} E^{* *} \\
F_{1}^{* *}=\frac{\alpha_{3}}{\left(\alpha_{3}+\mu_{F_{1}}+c_{\left.F_{1}\right)}\right)} A^{* *} \\
F_{2}^{* *}=\frac{\alpha_{3}}{\left(\mu_{F_{2}}+c_{F_{2}}\right)} F_{1}^{* *}
\end{array}\right.\right.
$$

em que $R_{M}>0$, e definido por

$$
R_{M}=\frac{\phi}{\left(\alpha_{1}+\mu_{E}+c_{E}\right)} \frac{\alpha_{1}}{\left(\alpha_{2}+\mu_{A}+c_{A}\right)} \frac{\alpha_{2}}{\left(\alpha_{3}+\mu_{F_{1}}+c_{F_{1}}\right)} \frac{\alpha_{3}}{\left(\mu_{F_{2}}+c_{F_{2}}\right)} .
$$

Biologicamente não faz significado falar que as populações do modelo (1) são negativas, portanto, $\Gamma=\left\{\left(E, A, F_{1}, F_{2}\right): E, A, F_{1}, F_{2} \geq 0\right.$ e $\left.0 \leq E \leq K\right\}$. Observe que o ponto $P_{1}$ tem significado biológico se $R_{M} \geq 1$. É biologicamente trivial, considerarmos $R_{M}=1$, em que $P_{1}=P_{0}$, representando um ponto de bifurcação transcrítica [3].

Assim como nos trabalhos de ( [8], [10]) utilizamos o critério de Routh-Hurwitz para avaliar a estabilidade dos pontos críticos do modelo (1). Desta forma, pode-se concluir que o ponto de equilíbrio $P_{0}$ é instável se $R_{M}>1$ e estável se $0<R_{M}<1$. O ponto de equilíbrio $P_{1}$ é instável se $0<R_{M}<1$ e estável se $R_{M}>1$.

No trabalho proposto por [1] foi adotado, por simplicidade, uma dependência linear entre os parâmetros entomológicos $\phi, \alpha_{1}, \alpha_{2}, \alpha_{3}, \mu_{F_{1}}$ e $\mu_{F_{2}}$ do modelo (1) e a pluviosidade acumulada semanalmente. A população do ciclo de vida do Ae. aegypti apresenta uma forte reação à presença de pouca pluviosidade, produzido relativamente muitos indivíduos. Contudo, para maiores quantidades de pluviosidade, a resposta da população não guarda a mesma intensidade. Portanto, neste trabalho, modelamos este comportamento por uma lei de potência, dada por:

$$
\boldsymbol{\pi}=\boldsymbol{\pi}_{\min }+\frac{\left(\boldsymbol{\pi}_{\max }-\boldsymbol{\pi}_{\min }\right)}{\left(\frac{1800}{52}-p_{\min }\right)^{r_{\pi}}}\left(p-p_{\min }\right)^{r_{\pi}}
$$

em que $\pi$ representa genericamente os parâmetros entomológicos $\phi, \alpha_{1}, \alpha_{2}, \alpha_{3}, \mu_{F_{1}}$ e $\mu_{F_{2}}$ do modelo $(1) ; r_{\pi} \in[00,8] ; p_{\min }$ representa a pluviosidade mínima da cidade em estudo; 
e $\left(\frac{1800}{52}\right)$ representa a pluviosidade média semanal da região de estudo.

\section{Material e Método}

Os dados amostrais de pluviosidade acumulada diariamente, são referentes às semanas epidemiológicas 12 a 52 do ano de 2009 e 1 a 51 do ano de 2010 da cidade de Sete Lagoas (Minas Gerais, Brasil) e foram obtidos do Instituto Nacional de Pesquisas Espaciais (INPE, Brasil) via Laborátorio de Ecologia Química da Universidade Federal de Minas Gerais LabEQ/UFMG.

Os dados amostrais de Índice Médio de Fêmeas Aedes - IMFA referentes ao período de estudo, foram obtidos da Ecovec SA via LabEQ/UFMG. Este índice corresponde à razão entre o número de fêmeas Aedes em fase de oviposição capturadas pela armadilha MosquiTRAP ${ }^{\circledR}$ e o número total de armadilhas instaladas na região de estudo.

O modelo (1) foi resolvido numericamente através do algoritmo de Runge-Kutta de quarta ordem utilizando o software MATLAB ${ }^{\circledR} 7.12 .0$ (R2011a) em um computador Intel(R) Core(TM) i5 2,5 GHz com 4GB de memória RAM. Adotamos as coordenadas do ponto de equilíbrio não trivial da primeira semana epidemiológica como valor das condições iniciais do modelo (1).

Os parâmetros $\alpha_{1 \max }, \mu_{E}, \alpha_{2}, \mu_{A}$ e $\mu_{F_{2} \text { min }}$ foram retirados de [4]; o parâmetro $\phi_{\max }$ foi retirado de [7]; os parâmetros $\phi_{\text {min }}, \alpha_{1 \text { min }}, K, \mu_{F_{1}}, \mu_{F_{2} \text { max }}$ e $\alpha_{3}$ foram estimados conforme ilustra a Tabela 1.

Tabela 1: Valor mínimo e máximo dos parâmetros entomológicos adotados no modelo (1).

\begin{tabular}{|c|c|c|c|c|c|c|c|c|}
\hline Taxas & $\pi_{\min }$ & $\pi_{\max }$ & Taxas & $\pi_{\min }$ & $\pi_{\max }$ & Taxas & $\pi_{\min }$ & $\pi_{\max }$ \\
\hline$\phi$ & 0,56 & 11,2 & $K$ & 1 & 1 & $\alpha_{1}$ & 0,01 & 0,5 \\
\hline$\mu_{E}$ & 0,01 & 0,01 & $\alpha_{2}$ & 0,06 & 0,16 & $\mu_{A}$ & 0,164 & 0,164 \\
\hline$\alpha_{3}$ & 0,2 & 1 & $\mu_{F_{1}}$ & 0,043 & 0,17 & $\mu_{F_{2}}$ & 0,057 & 0,17 \\
\hline
\end{tabular}

O valor da potência $r_{\pi}$ da equação (2) foi obtido através do Algoritmo Genético Real Polarizado - AGRP, proposto por [9]. Neste algoritmo foram adotados os seguintes parâmetros: 500 indivíduos na população, 300 gerações, taxa de crossover de $90 \%$ e taxa de mutação de $5 \%$. O problema de otimização consistiu em minimizar a função objetivo descrita como a distância entre as curvas de $I M F A$ e $F_{2}$ ao longo do horizonte de estudo. O valor ótimo da potência $r_{\pi}$ encontrado para os parâmetros $\phi, \alpha_{1}, \alpha_{2}, \alpha_{3}$, $\mu_{F_{1}}$ e $\mu_{F_{2}}$ é dado por $r_{\phi}^{*}=0,79985 ; r_{\alpha_{1}}^{*}=0,79993 ; r_{\alpha_{2}}^{*}=0,00004 ; r_{\alpha_{3}}^{*}=0,25874$; $r_{\mu_{F_{1}}}^{*}=0,79914$ e $r_{\mu_{F_{2}}}^{*}=0,79997$.

\section{Resultados e Discussões}

A evolução das populações do modelo (1) sem o uso de controle para a cidade de Sete Lagoas (Minas Gerais, Brasil), é apresentada na Figura 2. As populações e os dados de 
pluviosidade no horizonte de estudo foram normalizados para proporcionar uma métrica visual de comparação.
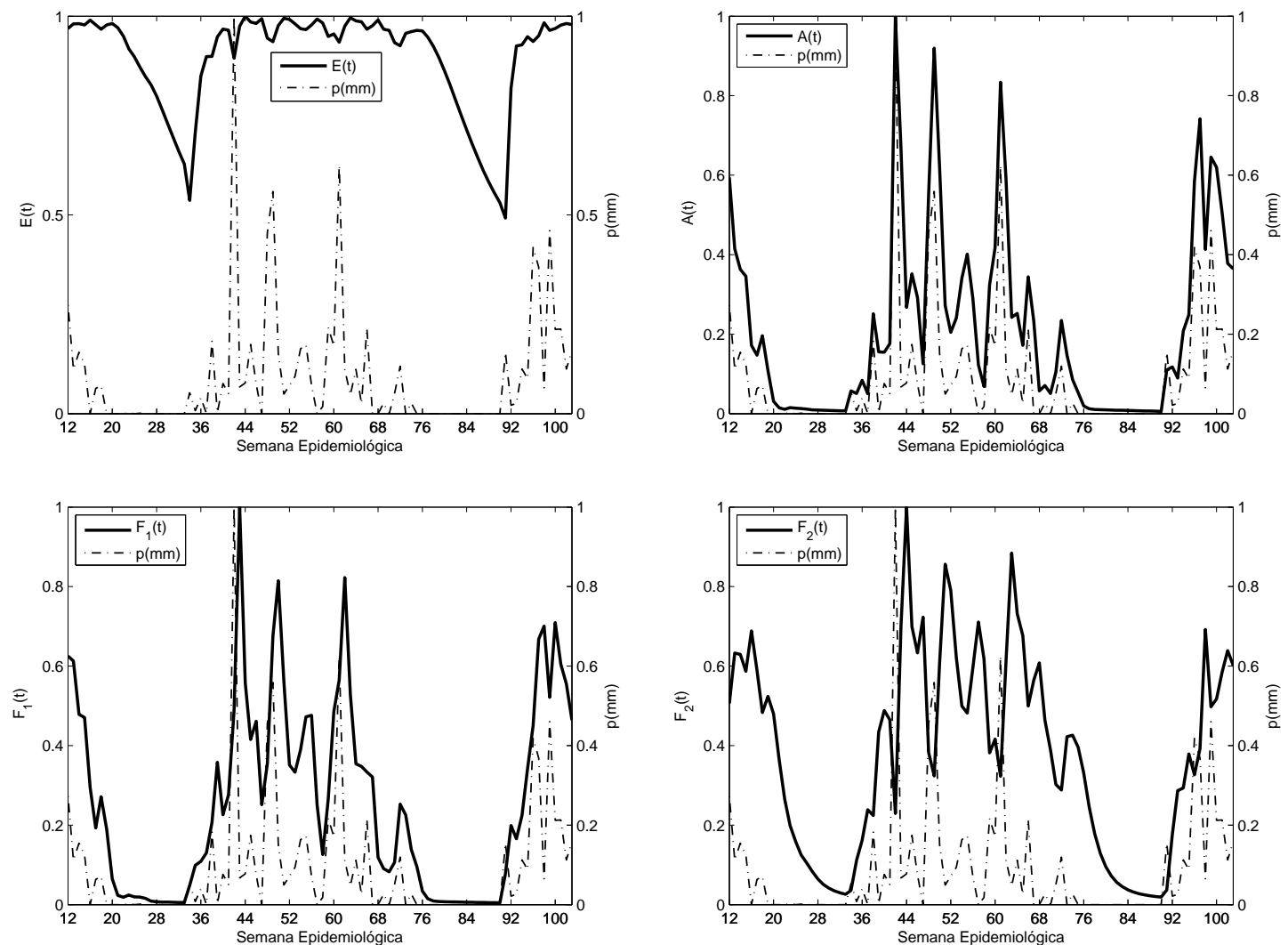

Figura 2: Evolução das populações $E, A, F_{1}$ e $F_{2}$ sem controle e da pluviosidade acumulada semanalmente, referentes às semanas epidemiológicas 12 a 52 do ano de 2009 e 1 a 51 do ano de 2010 da cidade de Sete Lagoas (Minas Gerais, Brasil).

A dinâmica da população $E$ segue a curva de pluviosidade com um atraso entre os picos de duas semanas. Observe que um pequeno período chuvoso é suficiente para manter esta população em níveis elevados e nos longos períodos de seca, como ocorrido entre as semanas epidemiológicas 26 a 33 e 75 a 90, esta população sofre um decaimento significativo. A população $A$ segue a curva de pluviosidade sem atraso entre os picos, evidenciando a forte influência da pluviosidade na dinâmica desta população. A população $F_{1}$ acompanha os índices pluviométricos com um atraso entre os picos de uma semana. A dinâmica da população $F_{2}$ segue a curva de pluviosidade com um atraso entre os picos de duas semanas. Nos longos períodos de seca, o decaimento da população $F_{2}$ é mais suave do que o decaimento da população $F_{1}$. A medição do atraso entre os picos da Figura 2 foi obtida via correlação cruzada.

A validação do modelo (1) ocorreu através da comparação qualitativa entre os resultados dos experimentos computacionais da população $F_{2}$ e os dados amostrais de IMFA 
no período de estudo, como ilustra a Figura 3.

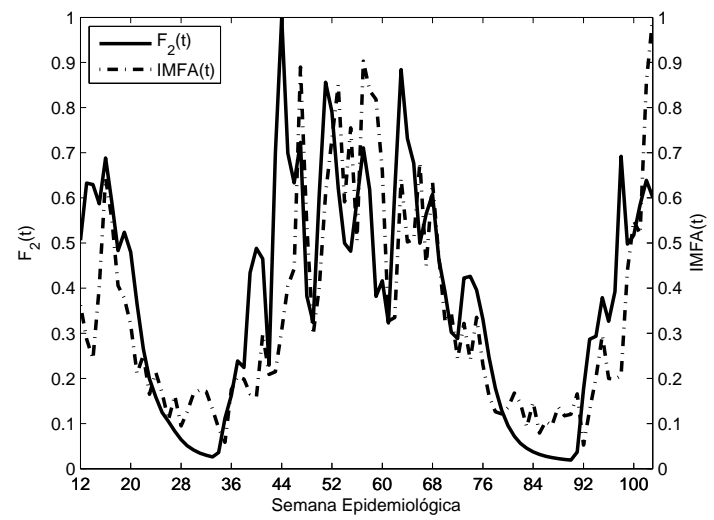

Figura 3: Comparação entre $F_{2}$ e $I M F A$ referentes às semanas epidemiológicas 12 a 52 do ano de 2009 e 1 a 51 do ano de 2010 da cidade de Sete Lagoas (Minas Gerais, Brasil).

A população $F_{2}$ acompanha os dados amostrais de $I M F A$ com um atraso entre os picos de uma semana, obtido via correlação cruzada. Observe que existem coincidências na posição dos picos e vales ao longo do horizonte chuvoso, porém o mesmo não foi observado no período de seca.

\section{Conclusões}

A evolução das populações do modelo (1) reproduziram a dependência dos parâmetros entomológicos do ciclo de vida do Ae. aegypti com a pluviosidade da cidade em estudo. A validação deste modelo pode ser considerada satisfatória no horizonte chuvoso como pode ser constatado pela proximidade entre $F_{2}$ e IMFA ao longo do horizonte de estudo. Como trabalho futuro pretende-se avaliar através de métodos de otimização, o melhor intervalo de tempo em que ações de controle deverão ser realizadas ao longo do ano. Este resultado poderá implicar em uma significativa redução dos gastos públicos no controle do vetor. Visando melhorar a adequação dos resultados amostrais de $I M F A$ com $F_{2}$, iremos incluir o efeito da inserção da população de machos do vetor e da umidade, juntamente com a pluviosidade e temperatura, nos parâmetros entomológicos do modelo (1).

\section{Agradecimentos}

Os autores expressam seus agradecimentos ao apoio financeiro da Fundação de Amparo a Pesquisa de Minas Gerais - FAPEMIG e da Coordenação de Aperfeiçoamento de Pessoal de Nível Superior - CAPES; as parcerias com o Laborátorio de Ecologia Química da Universidade Federal de Minas Gerais - LabEQ/UFMG e a Ecovec SA; e também do Centro Federal de Educação Tecnológica de Minas Gerais - CEFET-MG. 


\section{Referências}

[1] L. S. Barsante, Dependência entre pluviosidade e população de fêmeas Aedes aegypti grávidas descritas através de um sistema dinâmico não linear, Dissertação de Mestrado, Programa de Pós-Graduação em Modelagem Matemática e Computacional, Centro Federal de Educação Tecnológica de Minas Gerais, (2012).

[2] Casa das Ciências, Imagem do ciclo de vida do Aedes aegypti. Disponível em $<$ http : //imagem.casadasciencias.org/mosquito2.jpg >. Acesso em 24/05/15.

[3] F. S. Cordeiro, Modelo preditivo para a população de mosquitos Aedes aegypti sob influência de variáveis climáticas: temperatura e pluviosidade, Dissertação de Mestrado, Programa de Pós-Graduação em Modelagem Matemática e Computacional, Centro Federal de Educação Tecnológica de Minas Gerais, (2014).

[4] C. P. Ferreira e H. M. Yang, Estudo da transmissão da dengue entre os indivíduos em interação com a população de mosquitos Aedes aegypti, Tend. Mat. Apl. Comput, $4(3): 323-32,(2003)$.

[5] V. S. Gonçalves Neto e J. M. M. Rebêlo, Aspectos epidemiológicos do dengue no município de São Luís, Maranhão, Brasil, 1997-2002. Cad. Saúde Pública, 20(5):1424$31,(2004)$.

[6] J. Keating, An investigation into the cyclical incidence of dengue fever, Soc. Sci. Med.,53(12):1587-97, (2001).

[7] S. T. R. Pinho, C. P. Ferreira, L. Esteva, F. R. Barreto, V. C. Morato e Silva and M. G. L. Teixeira, Modelling the dynamics of dengue real epidemics, Philosophical Transactions of the Royal Society A: Mathematical, Physical and Engineering Sciences, 368(1933):5679-93, (2010).

[8] G. I. Poli e H. M. Yang, Modelo Matemático Aplicado para Imunologia de HIV, Tend. Mat. Apl. Comput, 7(2) 327-335, (2006).

[9] R. H. C. Takahashi, Otimização escalar e vetorial. Volume 2: Otimização escalar, (2007).

[10] R. C. A. Thomé, H. M. Yang, L. Esteva, Optimal control of Aedes aegypti mosquitoes by the sterile insect technique and insecticide, Mathematical Biosciences, 223:12-23, (2010).

[11] D. V. Viana e E. Ignotti, A ocorrência da dengue e variações meteorológicas no Brasil: revisão sistemática, Rev. Bras. Epidemiol., 16(2):240-56, (2013).

[12] World Health Organization, (2015). Disponivel em: http://www.euro.who.int/en/health-topics/communicable-diseases/vector-borneand-parasitic-diseases/dengue-and-chikungunya. Acessado em: 01/04/2015. 